\title{
Die Quellung von Rinde und Leitungsbahnen des Grosshirns als Funktion ihres chemischen Aufbaus.
}

\author{
Von \\ Arthur Weil.
}

(Aus dem physiologischen Institut der Universität Halle a. S.)

Mit 7 Textabbildungen.

(Eingegangen am 23. August 1919.)

Die Differenzicrung des embryonalen Gehirns, die allmähliche Trennung in graue und weisse Substanz, während der intrauterinen Entwicklung ist bis jetzt hauptsächlich nur Gegenstand histologischer Forschung gewesen. Seit Flechsig ${ }^{1}$ ) haben sich dic Anatomen mit Wrfolg bemüht, die Ausbildung der einzelnen Loitungsbahnen während der Markreifung zu verfolgen, um so ein klares Bild von dem zuerst unentwirrbar erscheinenden Durcheinander zu gewinnen. Es dauerte lange Zeit bis auch die physiologische Forschung angeregt wurde, dem. Zusammenhang zwischen der Markreifung und der Entwicklung der Gohirnfunktionen nachzugehen: es lag wohl an der ungenügenden Mothodik, wenn es den physiologischon Chemikern nicht gelang, die hiermit verbundenen Veränderungen der chemischen Struktur aufzudecken. Erst in neuerer Zeit boarbeiteten die Geschwister Koch die chemische Differenzierung des Gehirns während der Markreifung ${ }^{2}$ ). Sie beobachteten mit zunehmendem Alter eine Abnahme des Wassergehaltes und der Protcine, dafür eine Vermehrung der Lipoide während des Wachstums von Föten des Schweinos und der Albinoratten; nach Mansfeld und Liptak hält diese Zunahme dor Lipoide auf Kosten des Fiweisses auch noch während der extrauterinen Fintwicklung na ${ }^{3}$ ). - Die zunehmende Differenzierung im Wassergehalt dor grauen und weissen Substanz, die der Markreifung parallel goht, war schon den ersten Forschern bokannt, die sich mit der Chemie des Gchirns beschäftigten; man begrnügte sich aber stets mit der Feststellung der Tatsachen, ohne nach der Ursache dieses Unterschiedes zu forschen ${ }^{4}$ ).

1) P. Flechsig, Die Leitungsbahnen im Gehirn und Rückenmark. 1876.

2) W. Koch und M. L. Koch, Contributions to the chemical differentiation of the central nervous system. Journal of biolog. chem. vol. 14 p. 267, 281 und vol. 15 p. 423.1913.

3) G. Mansfeld und P. Liptak, Die quantitative Änderung der Hirnlipoide während der extrauterinen Entwicklung. Pflùger's Arch. Bd. 152 S. 68.1913.

4) Literatur vgl. A. Weil, Die Chemie des Gehirns. Zoitschr. f. d. ges Neurologie u. Psychiatrie Bd. 7 S. 1. 1913. 
Man muss über diese Gleichgültigkoit um so mehr staunen, wenn man sich daran erinnert, dass alle Theorien, welche die Funktion des Nervensystems zu erklären suchen, von Konzentrationsunterschieden zwischen den einzelnen morphologisch differenzierten Abschnitten ausgehen und je nach dem Stande der Forschung die verschiedensten Gesetze echter oder kolloider Lösungen auf die Reaktionen des lebenden Organismus übertragen, ohne zu prüfen, ob die Hauptphase dieser Systeme, das Wasser, überhaupt als Lösungsmittel in genügender Menge zur Verfügung steht, oder ob es als Quellungswasser gebunden ist. - Das Ziel der vorliegenden Arbeit war, zunächst einmal die Ursachen für den Untersehied in dem Wassergehalt der versehiedenen Teile des Nervensystems aufzudecken.

Das Wasserbindungsvermögen des Organismus ist ein reversibler Vorgang; das eingetrocknete Gewebe hat die Eigenschaft, in Wasser von bestimmter Temperatur wieder zu seiner ursprünglichen Form zurückzukehren; so nimmt zum Beispiel ein trockenes Stück Muskel unter bestimmten Bedingungen genau so viel Wasser wieder auf, wie es im lebenden Zustande gebunden hatte. Diese Eigenschaft, das Quellungsvermögen, kommt auch anderen, nicht organisierten Kolloiden zu, die sämtlich zur Gruppe der hydrophilen Kolloide gehören, und in deren Lösungen das Wasser so an die kolloiden Teilchen gebunden ist, dass es mit zur dispersen Phase wird und nur ein kleiner Rest frei bleibt ${ }^{1}$ ). Sie nehmen bei der Quellung „,die Flüssigkeit in das Innere des quellbaren Körpers auf unter Volumenzunahme, Festigkeitsabnahme und Zunahme der Elastizitätsgrenzen". ${ }^{2}$ ). Die meisten Kolloide gehen von dem Zustande der Quellung durch Wasseraufnahme allmählich in Lösungen über; so beobachtet man, dass ein Stiuck Lezithin in Wasser zunächst von den Rändern aus durchscheinend wird,., allmählich aufquillt, nach längerer Zeit in eine schleimige Flüssigkeit übergeht und nach einigen Tagen in eine klare, nicht dialysable Lösung umgewandelt wird. Auch andere Lipoide haben diese Eigenschaft; dagegen sind die eiweissartigen Substanzen meist nur begrenzt quellbar; so hat Gelatine bei Zimmertemperatur ein Quellungsmaximum, ebenso quillt geronnenes Serumeiweiss mit destilliertem Wasser immer nur bis zu einem bestimmten Grade. - Die von Hofmeister ${ }^{3}$ ) aufgestellten und von Ostwald ${ }^{4}$ ) u. a. bestätigten Anionenreihen für den hemmen-

1) R. Ḧ̈ber, Die physikalische Chemie der Zelle und der Gewebe S. 305. Leipzig 1914.

2) R. Zsigmondy, Kolloidchemie S. 115. Leipzig 1918.

3) F. Hofmeister, Untersuchungen über die Quellung. Arch. f. exper. Pathol. u. Pharmakol. Bd. 28 S. 210. 1910.

4) W. Ostwald, Über feinere Quellungserscheinungen bei Gelatine. Pflüger's Arch. Bd. 111 S. 581. 1906. 
Die Quellung von Rinde und Leitungsbahnen des Grosshirns usw. 23

den und fördernden Einfluss anorganischer Elektrolyte auf die Quellung wurden von Lenk ${ }^{1}$ ) nicht als allgemein gültig anerkannt, da or fand, dass sie nur für konzentrierte Lösungen galten, in stark gequollener Gelatine aber umgekehrt wirkten. Thre Wirkungen in kombinierten Lösungen addieren sich auch nicht, so dass antagonistische Tonen zusammen die Quellung stärker fördern können als das einzelne $\left.{ }^{2}\right)$. Die Wasseraufnahme hydrophiler Kolloide ist ferner abhängig von der Temporatur und der Spannung des Wasserdampfes. - Die wenigen Beispiele zoigen, wie kompliziert die Verhältnisse sind, unter denen die Quellung organisierter Gowebe zustande kommt; es ersoheint fast unmöglich, vergleichonde Cntersuchungen an ihnen vorzunehmen, da doch schon jede Zelle in ihrem Aufbau verschieden ist, in jedem Augenblick ihre Zusammensetzung ändert und der Einfluss der Elektrolyte durch Konzentrationsänderungen dauernd wechselt. - Mit Recht verlangt deshalb Katz, dass die Gesetze der Quellung nur an den einfachsten Körpern studiert werden dürfen, die aus einer homogenen. chemisch einheitlichen Verbindung bestehen sollen, welche don Einflüssen der Hysterese nicht unterliegt, und die frei von Porosität sein soll ${ }^{3}$ ).

Die wenigen bis jetzt erschienenen Arbeiton über dic Qucllung des Nervensystems haben diese einfachsten Grundbedingungen nicht berücksichtigt, sondern im Gegenteil noch an das Studium lebender, der Autolyse unterworfoner Substanz weitgehende theoretische Folgerungen geknüpft, dic im Widerspruch zu anderen, an nicht organisierten Geweben gewonnenen Erfahrungen standen. -..- Baucr, der den Einfluss von Säuren, Alkalion und Neutralsalzen auf das Rückenmark, die graue und weisse Substanz des Gehirns untersuchte ${ }^{4}$ ), glaubt auch die schon von Reichardt ${ }^{5}$ ) als Fehlerquellen erkannten Einflüsse der Autolyse vernachlaissigen zu dürfen, indem or sich auf eine Bemerkung Noll's stützt, ,dass das Zentralnervensystem sich wegen der spärlich in demselben vorhandenen Fermente nur langsam verändere, wenn es an der Luft liogengelassen wird". Er berücksichtigt aber nicht, dass er in Wasser, alkalischen und samren Lösungen quellen lässt, die die autolytische Zersetzung beschleunigen, und scine eigenen Versuchsergebnisse sind darum anch Beweise für den nicht zu vernach-

1) E.Lenk, Die Bedeutung der Flektrolyte für Quellungsprozesse. Bioch. Zeitschr. Bd. 73 S. 15. 1916.

2) Derselbe ebenda Bd. 73 S. 58.1916.

3) J. R. Katz, . Die Gesetze der Quellung. Kolloidch. Beihefte IX S. 1. 1917.

4) J. Bauer, Studien über Quellung von Nervengewebe. I. Mitt. Arb. aus dem neurolog. Inst. a. d. Wiener Cnivers. 1911 s. 111 u. 121. II. Mitt. mit Th. Ames, ebenda S. 226.

ə) M. Reichardt, Über die Cntersuchung des gesunden und kranken Gehirns mittels der Wage. Monatsschr. f. Psychiat. u. Neurol. Bd. 24. 1908. 
lässigenden Einfluss dieser Fehlerquellen ${ }^{1}$ ); so weist er auf die allmählich eintretende milchige Trübung des Quollungamittels hin, die Auflösung der gequollenen Substanz, die keine exakten Wägungen meht zulässt. - Seine Zeitkurve gibt am besten den Winfluss der. Autolyse wieder; das gecuollene Rückenmark zeigt kein Quellungsmaximum, die begrenzte Quellung des lebenden Organs geht allmählich in die kolloide Lösung über, dadurch, dass die angehäuften Stoffwechselendprodukte den ganzen molekularen Aufbau des biologischen Systems ändern. Als Beispiel hierfür erwähne ich die Angabe Fischer's ${ }^{2}$ ), dass Gelatine in Harnstofflösungen allmählich hydratisiert wird und das Gel zum Sol sich umwandelt. Der starke Sauerstoffverbrauch des lebenden Gehirns, auf den schon Ehrlich hinwies ${ }^{3}$ ), und den Winterstein mit seinen Schülern am überlebenden Organ unter allen Versuchsbedingungen feststellen konnte ${ }^{4}$ ), gibt einen Maassstab für die auch postmortal noch stattfindenden energischen oxydativen Reaktionen.

Ein Arbeiten mit frischen Organen kam aus allen diesen Gründen für mich aiso nicht in Frage; es handelte sich jetzt darum, Versuchsbedingungen zu tinden, die in bezug auf die Quellung dem lebenden Zustande möglichst gleichkamen, das heisst der Vorgang der Entquellung musste vollstandig reversibel sein bis zu dem Grade, welchen das dem Körper entnommene Organ besessen hatte. Als Versuchsmaterial benutzte ich Gehirne von Rindern, die wenige Stunden nach der Schlachtung verarbeitet wurden, unter Trennung in graue und weisse Substanz nach den schon wiederholt aufgestellten Grund. sätzen ${ }^{5},{ }^{6},{ }^{7}$ ).

Der Plan, nach dem lch bei meinex Arbeit, vorging, war folgender: Durch allmähliche Treonung der granen und weissen Substanz in die drei grossen Hauptoruppen: wasserlösliche Extraktivstofte, Lipoide und Eiweiss suchte ich den Einfluss festzustellen, welchen sie aufeinander in bezug aut die Quellung ausüben, um dann durch Umkehrung dieser Trennung, durch Mischen der einzelnen Komponenten

1) Bauer, 1. c. S. $112 \mathrm{f}$.

2) M. H. Fiseher u. A. Sykes, Über die nicht saure und nicht alkal. Hydratation des Proteins. Kolloid. Zeitschr. Bd. 16 S. 129.1915.

3) P. Ehrlich, Das Sauerstoffbedürfnis des Organismus. Berlin 1887.

4) H. W interstein, Über den Mechanismus der Gewebsatmung. Zeitsehr. f. allgem. Physiol. Bd. 6 S. 315. 1907.

5) E. A bderhalden u. A. Weil, Vergleichende Untersuchungen über den Gehalt der verschiedenen Bestandteile des Nervensystems an Aminosänren. I. Mitt. Zeitschr. f. physiol. Chemie Bd. 81 S. 207. 1912.

6) Dieselben, II. Mitt. ebenda Bd. 83 S. 425.1913.

7) A. Weil, Vergleichende Studien über den Gehalt verschiedenartiger Nervensubstanz an Aschebestandteilen. Ebenda Bd. 89 S. 349.1914. 
Die Quellung von Rinde und Leitungsbahnen des Grosshirns usw. 25

die erhaltenen Resultate zu kontrollieren. Als Maass für die Quellung sollte die maximale Menge Wasser gelten, die aus destilliertem Wasser von den getrockneten Substanzen aufgenommen wurde. - Zunächst galt es, die günstigsten Bedingungen für die Trocknung aufzufinden; als wasserentziehendes Mittel kam nur die Trocknung durch Wärme in Botracht; Entwässerung durch Alkohol, wie sie Koch vorschlägt ${ }^{1}$ ) oder durch Aceton nach Fränkel ${ }^{2}$ ) extrahiert gleichzeitig andere Substanzen. - Der bisher am meisten eingeschlagene Weg war dio Trokknung von Nervonsubstanz bei konstanter Temperatur, 50 bis 100 Grad im Trockensehrank oder im Faust-Heim'schen Apparat; man vergass aber dabei, dass durch den Einfluss des Sanerstoffes der Luft die labilen, ungesättigten Verbindungen, wie sie dic Lipoide enthalten, in ihrer Struktur vollständig verändert werden, so dass man eine von der lebenden Substanz völlig abweichende erhält. Vor allem sind os wohl die ungesättigten Fettsäuren der lezithinartigen Verbindungen, die leicht den Sauorstoff aufnehmen; absorbicrt doch die Ölsäure schon an der Luft leicht Sauerstoff, wobei sie zu niedrigeren Fettsäuren oxydicrt wird ${ }^{3}$. - - Diese Tatsache crklärt wohl auch die widersproshenden Befunde über die beiden Fettsäuren des Lezithins, die Diakonow als Stearinsäuren analysierte ${ }^{4}$ ), während nach Linnert ${ }^{5}$ ) die eine Komponente. Ölsäure ist. Alle Arbeiten, die so präparierte Niervensubstanzen zum Ausgangsmaterial haben, sind also sehr vorsichtig zu beurteilen. - Lässt man bei $50^{\circ}$ an der Luft getrocknete Nervensubstanz wicder quellen, so beobachtot man nach einiger Zeit, dass die Oberfläche sich mit eincr schleimigen Schicht überzieht, in die nach und nach die ganze Masse verwandelt wird, ein Zeichen dafür, dass der begrenzte Quellungszustand in die Lösung iibergeht. Im Vakuum getrocknete weisse und graue Substanz dagegen nimmt wieder das Volumen und die Beschaffenheit dos frischen Materials an, und der Quellungsgrad ist im Gegensatz zu der Iufttrockenen nicht herabyesetzt. Diese Art der Trocknung gestattet auch ein quantitatives Arbeiten beim Abtrocknen in Filtrierpapier, während bei der ersten Präparation ein grosser Teil der Substanz mit der schleimigen Oberfläche, abgelöst wird, abgesehen von der durchgreifenden Änderung der Struktur. - Die Tabelle I gibt oinige Beispiele für den Einfluss der verschiedenen Trocknungsarten auf die Wasserbindung. - Ich verfuhr hierbei folgendermaassen: Nach der präparativen Trennung

1) I. e. IT. Mitt. Bd. 14 S. 281.1913.

2) S. Fränke1, Über Lipoide. VI. Mitt. Bioch. Zeitschr. Bd.19 S. 265.1909.

3) Varrentrop. Beilstoin, Handbuch. I. Ergänzungsbd. S. 206.

4) Diak on ow, Zentralbl. f. d. mediz. Wissen 1868. Zitiert nach 5).

5) K. Linnert, Vergloichende chemische Hirnuntersuchungen. Bioch. Zeitschr. Bd. 26 S. 44. 1910. 
von grauer und weisser Substanz wurden mögichst gleiche Gewichtsmengen von etwa $1,5 \mathrm{~g}$ in kleine Wägegläschen gefüllt und entweder im Lufttrockenschrank oder in Porzellanvakuumexsikkatoren, die im Wasserbade bis auf eine Innentemperatur von etwa $60^{\circ} \mathrm{C}$. erhitzt und dauernd mit der Wasserstrahlpumpe rerbunden waren, bis zur Gewichtskonstanz getrocknet. Die von $\mathrm{Katz}^{1}$ ) angewandte Methode der Trocknung bis. $110^{\circ} \mathrm{C}$. im Exsikkator über Schwefelsäure gab keine anderen Resultate, wie ich mich durch Vorversuche überzeugen konnte. Die Difterenz im Gewichte der frischen und trockenen Substanz. ergab dann den Wassergehalt des lebenden Organs. - Zur Quellung wurde die zehnfache Menge destillierten Wassers hinzugefügt, die man aber beliebig ändern kann, da die Kcnzentration ohne Einfluss auf die Quellung ist (zehnfache und hundertfache Mengen gaben dieselben Werte). - Nach bestimmter Zeit wurden die gequollenen Stückehen auf faserfreiem Eiltrierpapier durch leisen Druck unter stetem Wechsel des Papiers so lange getrocknet, bis dieses kein Wasser mehr ansaugte. Eine aliquote Menge wurde wieder gewogen, bis zur Gewichtskonstanz getrocknet und ans der Differenz das Quellungswasser berechnet. - Ich befolgte hiermit die bis jetzt allgemein angewandte Methodik, ohne deren Nachteile, die in dem beim Trocknen ausgeübten wechselnden Druck liegen, zu verkennen; doch sind die hierdurch bedingten. Fehler, wie schon Hofmeister ${ }^{2}$ ) zeigte, so gering, dass sie vernachlässigt werden können. - Abweichend von der bisherigen Arbeitsmethode bestimmte ich die verschiedenen Kurvenpunkte nicht an ein und demselben Stück Substanz, da die Natur des Materials bei wiederholtem Abtrocknen und Wiederquellenlassen ein quantitatives Arbeiten verbietet. Tch arbeitete stets mit Serien ron sechs bis acht Gläsern, deren. Inhalt aus Gehirnteilen bestand, die möglichst einem eng umschriebenen Bezirk entstammten; die durch den verschiedenen chemischen Aufbau bedingte Fehlerquelle varkleinerte ich durch Zusammenstellung und Vergleich der verschiedensten Serien ein und desselben Gehirns. Es handelte sich für mich ja nicht darum, Gesetzmässigkeiten für die Quellung überhaupt aufzustellen, sondern das Verhalten zweier histiologisch scharf umschrïebener Gewebe. zu vergleichen. - Auch die Fehlerquelle, die etwa durch physikalische Faktoren, wie verschiedene Porosität bedingt sein könnte, kam nicht in Betracht, da es sich ja stets um dasselbe strukturell einheitliche Gewebe handelte.

Den Quellungsgrad definiere ich nach der allgemein üblichen Art als den Wassergehalt in Grammen pro $1 \mathrm{~g}$ trockener, quellbarer Substanz.

1) 1. c. S. 47.

2) 1. c. S. 217 . 
Die Quellung von Rinde und Leitungsbahnen des Grosshirns usw. 27

Für vergleichende Untersuchungen konnte ich diese Zahl, die ich nach Katz mit $i$ bezcichne, aber nicht verwenden, da sie nichts über die Anderung des Quollungsvermögens aussagt. Ich legte darum meinen Berechnungen und Kurven auch die Zahl $r$ zugrunde, welche Prozente des Wassergohaltes der lebendfrischen Substanz bedeutet.

\section{Tabolle I.}

Linfluss der Trocknung anf das Quellungsvermögen.

1: Quellungsgrad der lebenden Substanz (i).

2: Quellungsvermögen nach der Trocknang in Prozenten von 1 (r).

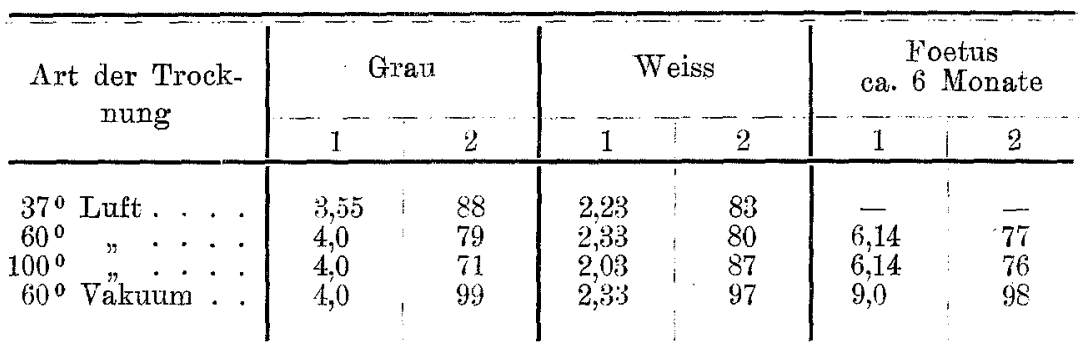

\section{Hemmung der Quellung durch die Hysteresis.}

Die von Hofmeister angewandte Methode des wiederholten Quellens und Entquellens von ein und demselben Gelatineplattchen, um vergleichende Messungen über die Wasserbindung in Salzlösungen und in roinem Wasser auszuführen, kann nicht ohne Einfluss auf das Quellungsvermögen sein. van Bemmolen hat diese Veränderung der Quellbarkeit von Gelen unter dern Einfluss wiederholter Quellungen und Fntquellungen beim Erhitzen eingehend studiert; danach muss man die hierbei anftretende Verminderung des Wasserbindungsvermögens, die Hysteresis, bei allen Versuchen in Rechnung setzen, besonders wenn man mit frisch hergestellten Präparaten arbeitet; im Laufe der Monate und Jahre wird diese Differenz geringer ${ }^{2}$. Auch bei den von mir benutzten Gehirnsubstanzen macht sich dieser Finfluss bemerkbar; am wenigsten tritt er bei don ursprünglichen Systemen in Erscheinung, grösser wird die Differenz, wenn man durch Extraktion mit verschiedenen Lösungsmitteln die Zusammensetzung ändert; es scheint aber, als ob bei den unveränderten Substanzen nach etwa, drei, bei den nur mit Benzol extrahierten nach etwa sechs aufeinander folgenden Quellungen und Entquellungen ein Gleichgewichts\%ustand erreicht würde. - In der 'Tabelle IT habe ich diese Kinflüsse an Beispielen zusammengestellt. --- Die praktische Folgerung, die ich daraus zog, war, dass ich jede Serie höchstens für nur zwei Quellungsversuche

1) J. M. van Bemmelen, Die Absorption S. 266 f. Dresden 1910. 
henutyte und meistens nur den zweiten meinen Kurven zugrunde legte, um einheitliche, vergleichbare Werte za erhalten. - Die Erscheinung der Steigerung des Quellungsvermögens während der zweiten Quellung worde ich weiter unten zu erklären versuchen (S. 36).

\section{Tabelle II.}

Einfluss der Hysteresis auf das Quellungsvermögen.

Zahlen $=r=$ Prozente des Wassergehaltes der lebenden Substanz.

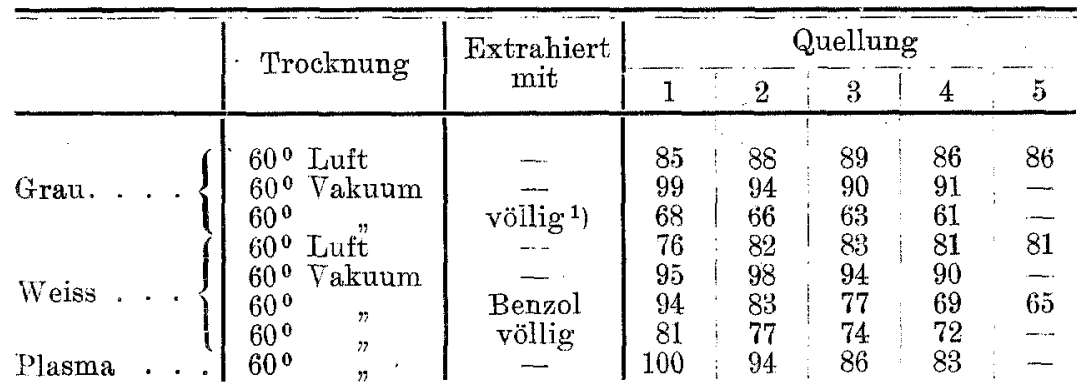

Zum Vergleich zog ich ein beliebiges anderes Gelsystem des Körpers heran; ich wählte Plasmaeiweiss, das ich durch Hitzekoagulation von Oxalat-Rindsrblut gewonnen hatte und durch mehrtägige Dialyse mit oft erneucrtem Wasser von den löslichen Beimengungen befreite; die Trocknung. war dieselbe wic beim Gehirn. - Dio verschiedenen Quellungen verglich ich untereinander, indem ich den Wassergehalt nach der ersten mit 100 bezeichnete; die Tabelle II zeigt die Abnahme des Quellungsvermögens im Gegensatz zu dem nur wenig veränderten Gewebe. - Man kann das geronnene Plasma mit demsclben Recht als ,homogene:" Substanz verwenden wie Fibrin, Elastin, Serumalbumin und andere aus dem lebenden Organismus gewonnenen Gele; denn wir wissen von keinem, ob os chemisch einheitliche Körper sind, und der wechselnde Aschegehalt, die noch nicht entschicdene Frage über die Boimengungen von anderen Kolloidon gibt allen Systemen nur einen bedingten Wert für die Aufstellung von Quellungsgesetzen. Iie Tatsache, dass Jibrin, Elastin u. a. alle möglichen Kolloide, zum Beispiel Pepsin, adsorbieren. können, spricht im Gegenteil dafür, dass auch bei der Koagulation aus dem lebenden Gewebe sich diese Gele als Adsorptionsverbindungen aus den verschiedensten Substanzen bilden $\left.{ }^{2}\right)$.

\section{Die fraktionierte Extraktion.}

Nachdem ich so die beiden wichtigsten Finflüsse auf die Quellung des getrockneten Gehirns studiert hatte, konnte ich an die Ausführung

1) Tach Extraktion mit Wasser, Petroläther, Benzol und Alkohol; vgl. S. 26.

2) E. Abderhalden, Physiologische Chemie. Bd. I S. 469. 1914. 
Die Quellung von Rinde und Leitungsbahnen des Grosshirns usw. 29

des eigentlichen Planes meiner Arbeit gehen, durch Trennung dex. einzelnen Bestandteile ihre gegenseitigen Beziehungen zu crforschen.

Die Kenntnisse über den chemischen Aufban des Gehims sind noch sehr gering; als sicher feststehende Tatsachen kann man wohl nur annehmen, dass neben Eiweiss eine Gruppe von Verbindungen als Hauptbestandteil vorkommt, die die gemeinsame Figenschaft haben, sich im Gegensatz zu den Protoinen in bestimmten organischen Flüssigkeiten zu löscn. Seit Thudichum ${ }^{1}$ ) haben sich unzählige Forscher bemüht, die chemische Struktur dieser Körper zu onträtseln; bis jetzt ist es in keinem Falle gelungen, die hypothetischen Annahmen sicher zu stellen, auch nicht die der am besten isoliorten Cholesterin und Lezithin; die Grün'sche Synthese führte nur zu optisch inaktiven Distearinderivaten oder Lezithinen mit zwei anderen gesättigten Fettsäurcn; die Darstellung der natürlichen Verbindung, die daneben noch Ölsäure enthält, gelang noch nicht ${ }^{2}$ ). - - Die Chemie der Lipoide steht heute etwa auf dem Standpunkt, den die . Kiweisschemic vor den Forschungen Emil Fischer's erreicht hatte: man kann wohl bestimmte Gruppen durch fraktionierte Fällung und Extraktion isolicren, man hat auch schon einzclne einheitliche Verbindungen von konstanter analytischer Zusammensetzung zu gewinnen gelernt; aber die Frage, $o b$ es sich bei diesen um Spaltprodukte oder natürlich vorkommende Verbindungen handelt, und die Klärung der Konstitution der einzelnen Fraktionen ist nicht gelöst, wie der noch immer noch nicht geschlichtete Streit um das Protagon beweist ${ }^{3}$ ). - Auch die fraktionierte Extraktion, wie sie Fränkel ausarbeitete, führte unter den verschiedensten Versuchsbedingungen richt immer zu einheitlichen Ergebnissen ${ }^{4}$ ). - -. So ist besonders der Einfluss der Trocknung zu beachten; eigene Untersuchungen zeigten mir aber, dass man beim Trocknen im Vakuum immer annähernd gleiche Resultate erhält, wenn man stets erschöpfend extrahiert; die Erklärung für die wechselnde Ausbente bei Tufttrocknung habe ich bereits gegeben (S. 23). - In der Ausführung hielt joh mich an die von Fränkel aufgestellten Vorschriften, indem ich nacheinander mit Wasser, Aceton, Petroläther, Benzol und Alkohol extrahierte; die Schlussextraktion mit Äthei unterliess ich, da beim Rindergehirn keine nennenswerten Mengen mehr in Lösung gehen; dagegen behandelte ich zuerst mit $W$ asser, um die löslichen anorgani

1) J. I. W. Thudichum, Die ehemische Konstitution des Gehirns des Menschen und der Tiere. Tübingen 1901. Vgl. hier auch ältcre Literatur.

2) A. Grün u. F. Kade, Zur Synthese der Lezithine. Ber. d. D. Chem. Ges. Bd. 45 S. 3367. 1912.

3) O. Rosenheim u. M. Chr. Tebb, Die Nichtexistenz des sogenanaten Protagons im Gehirn. Bioch. Keitschr. Bd. 25 S. 151. 1910.

4) S. Fränkel, Vergleichende chemische Gehirnuntersuchungen. Bioch. Zeitschr. Bd. 19 S. 265.1909. 
schen Bestandteile und Stoffwechselprodukte zu entfernen. Vom Aceton werden hierbei hauptsächlich Cholesterin und -ester sowie einzelne Phosphatide aufgenommen; in den Petroläther gehen ungesättigte Phosphatide, die in die Kephalinfraktion und Myelin mit Monophosphatiden getrennt worden können; in das Benzol Corebroside, P. und S haltige Galaktoside, und der Alkohol schliesslich nimmt Cerebroside und gesättigte Phosphatide (Sphingomyelin) auf.

Für moine Untersuchungen kam es zunächst nicht darauf an, wie diese einzelnen Fraktionen chemisch zusammengesetzt sind, sondern das Interesse beschränkte sich darauf, festzustellen, wie sich das Quellungsvermögen nach den einzelnen Fxtralktionen verhiclt. -... Die Tabelle III gibt zunächst einen Utberblick über dio Extraktverluste der grauen und woissen. Substanz vom Gehirn eines etwa dreijährigen Rindes, die nach jeder Extraktion bei $60^{\circ} \mathrm{C}$. im Valzum getrocknet wurden; daneben führe ich zum Verglcich dje Zahlen Fränkels tür ein nicht in diese beiden Bestandteile getrenntes menschliches Gehirn an.

Tabelle III.

\section{Fraktionierte Extraktion des Gehirus.}

Verluste in Prozenten der extrahierten Substanz (1) und des Ausgangsmaterials (2).

\begin{tabular}{|c|c|c|c|c|c|c|c|c|}
\hline \multirow{2}{*}{$\begin{array}{c}\text { Nach Extraktion } \\
\text { mit }\end{array}$} & \multicolumn{2}{|c|}{ Grau } & \multicolumn{3}{|c|}{ Weiss } & \multicolumn{3}{|c|}{$\begin{array}{c}\text { Mensch } \\
\text { (nach Fränkel) }\end{array}$} \\
\hline & 1 & 2 & $i$ & 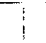 & 2 & 1 & & 2 \\
\hline Wasser & 13 & 13 & 6 & 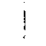 & 6 & - & 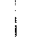 & - \\
\hline Aceton & 19 & 17 & 17 & & 16 & 10,96 & & 10,96 \\
\hline Petroläther. . & 22 & 16 & 44 & & 34 & 31,25 & & 27,84 \\
\hline Benzol . . . & 6 & 3 & 21 & $i$ & 9 & 22,1 & $!$ & 13,53 \\
\hline Alkohol. & 12 & 6 & 14 & & 3 & 13,1 & & 6,26 \\
\hline Äther. & - & $-\cdots$ & 一 & & $\ldots$ & 2,2 & & 0,92 \\
\hline Rest : . . . & - & 45 & -- & & 32 & - & $!$ & 31,63 \\
\hline
\end{tabular}

Einen zweiten Versuch stelle ich zum besseren Vergleich mit den folgenden Qucllungsversuchen graphisch dar; die Extraktion erfolgte hierbei mit Wasser, Aceton., Benzol, Alkohol und Ẍther. Die Ordinate der Kurve gibt die Extraktverluste in Prozenten des Ausgangsmaterials an; die Abszisse bezeichnet das angewandte Extraktionsmittel. -- Die Kurve zeigt den höheren Gehalt der grauen Substanz des lufttrockenen Rindergehirns an Acetonextrakt gegenüber der weissen und das Cberwiegen der benzollöslichen Besţandteile in den letzteren; in beiden tehlen die ätherlöslichen Reste. - Im Trockenschrank bei $60^{\circ} \mathrm{C}$. getrocknet. 
Die Quellung von Rinde und Leitungsbahnen des Grosshirns usw. 31

Gleichzeitig mit den Extraktverlusten analysierte ich die anorganischon Bestandteile der einzelnen Mralrtionen. Die Methodik war diesolbe wie die früher von mir angewandte ${ }^{1}$ ). Unter Berücksichtigung der Addition der Fehler durch die Untersuchung der verschiedenen

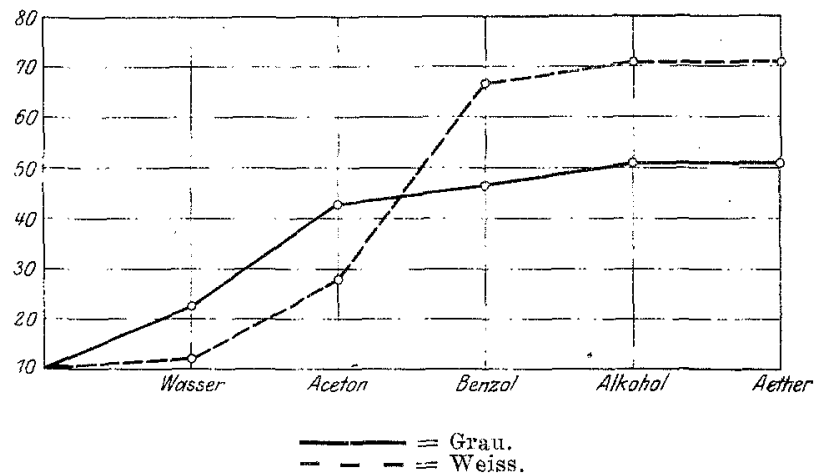

Abb. 1. Fraktionierte Fixtraktion von grauer und weisser Substanz.

getrennten Bestandtoile und der zur Verfügung stehenden kleinen Mengen - ich ging von $102 \mathrm{~g}$ frischor grauer und $175 \mathrm{~g}$ frischor weisser Substanz aus - stimmen die Endsummen mit den schon früher veröfentlichten Zahlen überein; nur der Ca- und $\mathrm{Mg}$-, sowie der Cl-Gehalt der weissen Substanz bleibt hinter dem erüheren Durchschnitt zurück und kommt der Kusammensetzung des Rückenmarkes näher.

\section{Tabolle IV.}

\section{Anorganische Bestandteile und Stickstoff in den Extraktfraktionen.}

Spalte 1: Gehali von 1000 g Trockensubstanz; Spalte 2-6: Verteilung auf die einzelnen Extrakte und den nicht extrahierbaren Rest in Prozenten von 1

A. Graue Substanz. Wassergehalt $80 \%$.

\begin{tabular}{|c|c|c|c|c|c|c|}
\hline & $\begin{array}{l}g \\
1 \\
\end{array}$ & $\begin{array}{c} \\
\text { Wasser } \\
2\end{array}$ & $\begin{array}{c}\text { Aceton } \\
8 \\
\end{array}$ & $\begin{array}{c}\text { Benzol } \\
\text { ju.Petrol- } \\
\text { ather } \\
4\end{array}$ & $\begin{array}{c}\text { Alkohol } \\
5\end{array}$ & $\begin{array}{c}\text { Rest } \\
6\end{array}$ \\
\hline 1. $\mathrm{Ca} . .$. & 0,860 & 40,5 & - & 2,9 & -- & 56,5 \\
\hline 2. $\mathrm{Mg} \cdot \ldots$ & 0,716 & 47,0 & $\ldots$ & 58,0 & - & $\ldots$ \\
\hline 3. P... & 12,45 & 24,5 & 2,8 & 52,2 & $\check{5}, \check{5}$ & 15,0 \\
\hline 4. $\mathrm{S} . .$. & 3,21 & 20,5 & 2,0 & 10,8 & 7,5 & 59,4 \\
\hline 5. Cl..... & 7,40 & 67,0 & - & 3,4 & 24,0 & 5,4 \\
\hline 6. N... & 81,44 & 8,2 & 2,4 & 11,7 & 2,8 & 75,0 \\
\hline
\end{tabular}

1) A. W eil, 1. c. S. 356. 
B. Weisse Substanz. Wassergehalt $69 \%$.

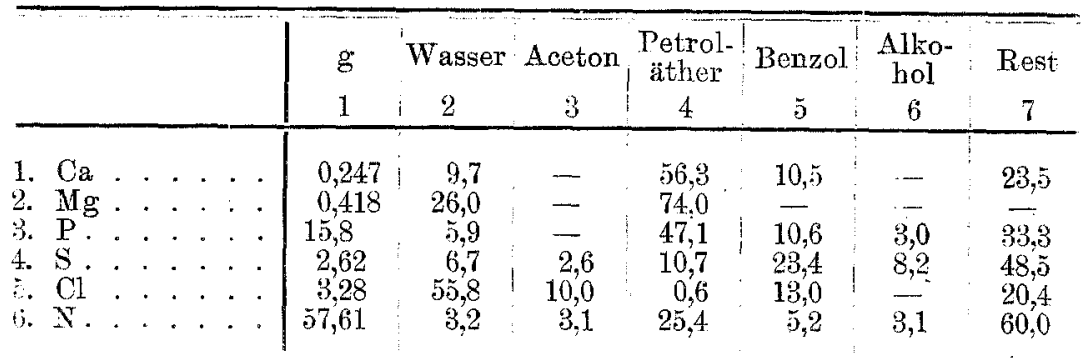

Wio sehr diese Verteilungszahlon auch wieder von der Art der I'rocknung abhängen, zeigten mir die Analysen eines vollständig extrahierten Gehirns, das nach den cinzelnen Extraktionen im Thermostaten bei $60^{\circ} \mathrm{C}$. getrocknet war. - Ich stelle die auf $1000 \mathrm{~g}$ der extrahierten Substanz berechneten Zahlen mit den entsprechenden, aus TabelleIV A, 6 und $B, 7$ berechncten zusammen und füge gleichzeitig die Analysen eines Gehirns von einem Fötus im zirka siebenten Monate hinzu.

Tabelle V.

$1000 \mathrm{~g}$ trockene extrahierte Substanz enthalten:

\begin{tabular}{|c|c|c|c|c|c|}
\hline & $\begin{array}{l}\text { Grau } \\
\text { luftt }\end{array}$ & $\begin{array}{l}\text { Woiss } \\
\text { ken }\end{array}$ & $\begin{array}{l}\text { Grau } \\
\text { IV A }\end{array}$ & $\begin{array}{l}\text { Weiss } \\
\text { IV B }\end{array}$ & Foetus \\
\hline 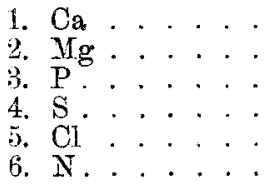 & $\begin{array}{r}0,26 \\
1,54 \\
2,20 \\
2,4 \\
129,0\end{array}$ & $\begin{array}{c}0,38 \\
0,53 \\
2,93 \\
3,5 \\
124,0\end{array}$ & $\begin{array}{r}1,04 \\
4,00 \\
4,09 \\
0,85 \\
131,0\end{array}$ & $\begin{array}{c}0,18 \\
-2 \\
16,6 \\
4,02 \\
2,1 \\
109,0\end{array}$ & $\begin{array}{c}0,665 \\
1,38 \\
11,46 \\
1,47 \\
6,93 \\
87,6\end{array}$ \\
\hline
\end{tabular}

Zunächst einigo Bemerkungen über die Bedeutung dioser Tabellen:

Lange Zeit hatte man angenommen, dass die stets bei der Analyse von Proteinen gefundenen Aschebestandteile als Vorunreinigungen aufzufassen seien, die in keiner Beziehung zu dew molckularen Aufbau ständen. Die sieh aber immer mehr häufenden Beobachtungen äber den Einfluss der Neutralsalze auf Kolloide zwangen zu der Annahme, dass diesem steten Vorkommen von "Verunreinigungen" eine bestimmte Gesetzmässigkeit zugrunde liegen müsse. Robertson, der die Proteine als amphotere Elcktrolyte betrachtete, nahm eine salzartigo Bindung mit den einzelnen, in wässeriger Lösung dissoziierten Ionen dor Neutralsalze an $\left.{ }^{1}\right)$; andere fassen diese Bindungen als Adsorptionserseheinungen auf, also als eine Funktion der Oberfläche, wie Ostwald ${ }^{2}$ ); wieder

1) T.B. Roberts on, Die physikalische Chemie der Proteine. Dresden 1912.

2) 1. c. 
Die Quellung von Rinde und Leitungsbahnen des Grosshirns usw. 33

andere im Sinne van Bemmelen's ${ }^{1}$ ) nehmen bei den Gelen eine Absorption mit dem Quellungswasser in die Wasserhüllen der Nägelischen Micellen oder die Wabenhohlräume Bütschli's an. - Auch die Lipoide enthalten ebenso wie die Proteine oft bedeutende Mengen anorganischer Salze. So erwähnt Fränkel ${ }^{2}$ ), dass Kephalin in Petroläther bis $17 \%$ seines eigenen Gewichtes Glaubersalz zu binden vermag, und auch Thudichum unterstreicht die stete, "Verunreinigung" Fon Lezithin mit Kalium- und Calciumsalzen ${ }^{3}$ ). - Diese Tatsachen machen es verständlich, dass ein grosser Prozentsatz von $\mathrm{Ca}$ und $\mathrm{Mg}$ an die Petroläther- und Benzolfraktion gebunden ist; das gleichzeitige Auftreten von $\mathrm{Cl}$ spricht vielleicht für eine Adsorption dieses Ions an Lipoide. Die Verteilung von $\mathrm{P}$ und $\mathrm{S}$ geht parallel mit dem Gehalte an extrahierten Substanzmengen (Tab. III); der grössere Gehalt der grauen Substanz an wasserlöslichem $\mathrm{P}$ und $\mathrm{S}$ beruht auf dem gesteigerten Stoffwechsel der Rindenzellen, deren Sauerstoffverbrauch nach Ehrlich ja auch etwa fünfmal so gross ist wie der anderer Körpergewebe. - Der Stickstoff der Benzol- und Petrolathertraktion ist hauptsächlich an Lipoide gebunden; das Verhältnis zum Phosphor dieser Fraktion in der grauen Substanz deutet schon darauf hin: die Zahlen 52,2 und 11,7 verhalten sich wie die Atomgewichte 2 P:N. Der Gehalt der extrahierten Substanz an $\mathrm{N}$ und $\mathrm{S}$ entspricht ungefähr dem Prozentgehalt, wie er für die meisten Proteine gefunden wurde; die für Grau und Weiss übereinstimmenden Zahlen lassen vermuten, dass die Eiweisskörper in beiden dieselben sind. Eine Stütze für diese Anschauung ist auch die von Abderhalden und mir gefundene Übereinstimmung in dem Gehalt an den verschiedenen Aminosäuren ${ }^{4}$ ); doch trotz dieser elementaren Übereinstimmung könnten noch grosse Strukturunterschiede vorhanden sein. Eine dritte Stütze für meine Hypothese gibt die Entwicklungsgeschichte des Nervensystems. Wenn. man mit His, Kölliker u. a. den Achsenzylinder als Ausłäufer des Protoplasmas der Ganglienzelle betrachtet oder mit Hensen als eine kettenartige Verbindung durch Teilung entstandener neuer Zellen ${ }^{5}$ ), so liegt der Schluss nahe, dass die chemische Zusammensetzung des Protoplasmas der Zelle und ihres Fortsatzes dieselbe sein muss, denn das ist ja eben die Eigenart der lebenden Substanz, auch ihre chemische Struktur bis anf die kleinste Einzelheit bei der Teilung weiter zu vererben. Die Differenzierung während der Markreifung würde dann

1) 1. c. S. 422 .

2) S. Fränkel u. E. Neubauer, Über Lipoide. Bioch. Zeitschr. Bd. 21 S. 321.1909 .

3) 1. c. S. 131 น. 312 ,

4) 1. c. II. Mitt. S. 427.

5) Vgl. O. Hertwig, Entwicklungsgeschichte des Menschen und der Wirbeltiere S. 580 f. Jena 1910.

Pflüger's Archiv für Physiologie. Bd. 179. 
darin bestehen, dass um diesen einheitlichen Kern eine Hülle aus Lipoiden gelagert würde.

Auffallend ist in Tabelle $V$ der hohe Gehalt der völlig extrahierten, nicht durch die Lufttrocknung veränderten grauen und weissen Substanz an Phosphor. - Da die Proteine selbst keinen enthalten und die vorhandenen Mengen von Kationen zur Bindung als Phosphate nicht genügen, muss man annehmen, dass auch nicht in den üblichen Flüssigkeiten lösliche ,Lipoide" vorhanden sind, oder dass diese Lipoide fester an das Eiweiss verankert sind als die grosse Menge der extrahierbaren. Für diese letzteren besteht wohl kaum eine Bindung als Lipoproteine im Sinne Bondi's ${ }^{1}$ ); die Verkettung der von ihm dargestellten Verbindungen von Aminosäuren mit Fettsäuren ist so fest, dass eine Trennung durch einfache Extraktion nicht anzunehmen ist. Man kann diesen Tatsachen viel eher gerecht werden, wenn man annimmt, dass die gegenseitige Anziehung ron Tipoid- und Eiweissteilchen dadurch erfolgt, dass sie durch die adsorbierten Salze eine verschiedene, entgegengesetzte, elektrische Ladung erhalten. Jedes Protein- und Lipoidteilchen würde dann mit dem Quellungswasser und den ElektroIyten ein System für sich bilden. - Die allgemeinere Anschauung, die auch die heutigen Vorstellungen über die Vorgänge bei den Immunitätsreaktionen beherrscht $\left.{ }^{2},{ }^{3},{ }^{4}\right)$, geht dahin, dass die Lipoidteilchen direkt an die Oberfläche der Eiweissteilchen gebunden sind oder umgekehrt.

Bei der Extraktion könnten dann diese Bindungen so gesprengt werden, dass die organischen Lösungsmittel das Wasser von der Oberfläche der Lipoidteilchen verdrängen und damit das Potential gegen die Proteinteilchen so verändern, dass keine gegenseitige Anziehung mehr erfolgt. Voraussetzung hierfür ist, dass die Lyrotropie der Lipoide im Sinne Freundlich's ${ }^{5}$ ) geringer ist als ihr ,Anziehungsvermögen " für die organischen Lösungsmittel. - Für anorganische Elektrolyte, Aminosäuren und Kohlehydrate ist ein solches gegenseitiges Verdrängungsvermögen von der Oberfläche adsorbierender Kohleteilchen bereits nachgewiesen und auch für kolloide Systeme wahrscheinlich

1) S. Bondi, Über Lipoproteide und die Deutung der degenerativen Zellverfettung. Bioch. Zeitschr. Bd. 17 S. 542. 1909.

2) H. Handovsky.u. R. Wagner, Physikalisch-chemische Eigenschaften von Lezithinemulsionen und Lezithineiweissgemischen. Bioch. Zeitschr. Bd. 31 S. 43. 1ø11.

3) E, P. Pick u. O. Schwarz. Über die Beeinflussung der Antigenwirkung dureh Lezithin. Bioch. Zeitschr. Bd. 15 S. 453. 1909.

4) H. Sachs u. W. Georgi, Serodiaguostik der Syphilis mittels Ausflockung. Theorie und Literatur vgl. W. Georgi, Studien über Serum-. ausflockung bei Syphilis. Bioch. Zeitschr. Bd. 93 S. 16. 1919.

5) H. Freundich, Kapillarchemie S. 54. 1909. 
Die Quellung von Rinde und Leitungsbahnen des Grosshirns usw. 35 gemacht $1,2,3)$. - Auch die entquellende Wirkung der verschiedenen Salze kann man in diesem Sinne als Verdrängung des Wassers von der Oberfläche der gequollenen Teilchen auffassen.

Für den Rest der im Extraktionsrückstand verbleibenden, nicht in den üblichen organischen Lösungsmitteln löslichen, Lipoide " bliebe dann eine festere, durch chemische Affinitäten bedingte Verkettung im Sinne Bondi's anzunehmen, die dureh Veränderung der chemischen Struktur, wie sie beim Erhitzen in Gegenwart von Sauerstoff stattfindet, gesprengt wird (Tab. V, Grau und Weiss lufttrocken).

Nacb jedem Stadiam der fraktionierten Extraktion prüfte ich das Quellungsvermögen durch Feststellen des Quellungsmaximums nach 24 Stunden bei 18-20 C. - Tab. VI gibt die Zahlen für ein bei $60^{\circ} \mathrm{C}$. an der Luft getrocknetes Gehirn eines zirka fünfjährigen Ochsen und die entsprechenden Werte für ein im Vakuum getrocknetes Rindergehirn. Kurve II stellt die Quellungsunterschiede für ein vakuumgetrocknetes Gehirn graphisch dar.

\section{Tabelle VI.}

Quellungsmaximum nach fraktionierter Extraktion.

Quellungsgrad $r=$ Prozente des Wassergehaltes der Iebenden Substanz.

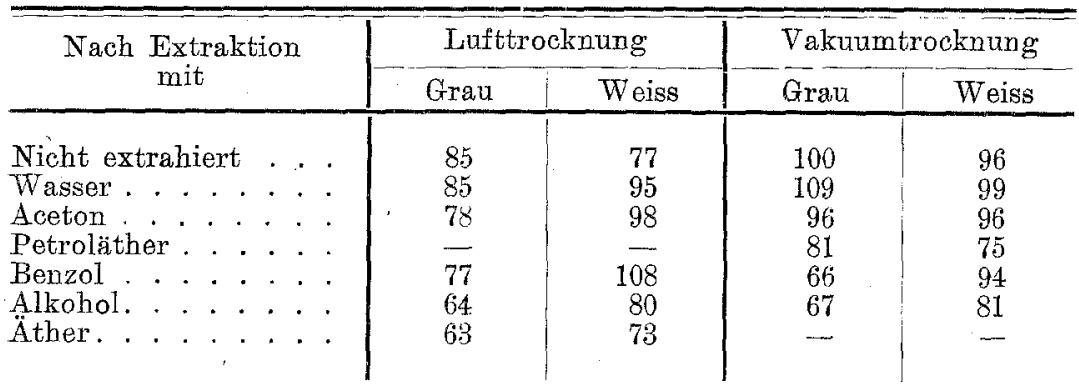

Die für die vergleichenden Untersuchungen hauptsächlich in Betracht kommende. Kurve II zeigt zunäshst einen Anstieg des Quellungsvermögens nach der Extraktion mit Wasser. Wir haben hier dieselbe Erscheinung wie in Tabelle II nach der zweiten Quellung. Die in Lösung befindlichen, mit Wasser extrahierbaren Substanzen, also anorganische Salze, Stoffwechselendprodukte, Reservestoffe der Zelle usw. haben eine hemmende Wirkung auf die Quellung, so dass nach

1) E. Abderhalden u. A. Fodor, Forschungen über Fermentwirkungen. Fermentforschungen Bd. II S. 74-102, 151-166, 211-224. 1918.

2) E. Eichwald u. A. Fodor, Die physikalisch chemischen Grundlagen der Biologie S. 222. Berlin 1919.

3) P. R ona u. L. Michaelis, Über Adsorption von Elektrolyten durch Kohle. Bioch. Zeitschr. Bd. 94 S. 240. 1919. 
ibrer Entfernung das Wasserbindungsvermögen steigt. Den Beweis hierfür liefert die Umkehrung dieses Vorganges, indem man die wässe-

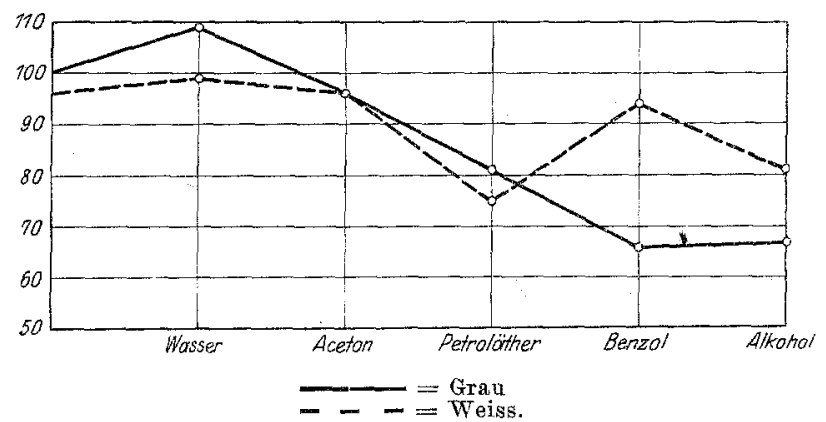

Abb. 2. Abszisse $=$ Extraktionsmittel. Ordinate $=r=$ Prozente des ursprünglichen Wassergehalts.

rigen Extrakte wieder zu der extrahierten Gehirnsubstanz hinzufügt. Die Tabelle VII gibt die erhaltenen Resultate wieder.

Tabelle VII.

Hemmung der wässerigen Extrakte auf die Quellung. (Lufttrocknung.)

\begin{tabular}{|c|c|c|c|c|c|}
\hline \multirow[t]{2}{*}{ Substanz } & \multirow{2}{*}{$\begin{array}{l}\text { Wasser- } \\
\text { gehalt } \\
\text { (lebend) in } \\
\text { Prozenten }\end{array}$} & \multicolumn{2}{|c|}{$\begin{array}{l}\text { Quellung der extra- } \\
\text { hierten Substanz }\end{array}$} & \multicolumn{2}{|c|}{$\begin{array}{l}\text { Quellung der extra- } \\
\text { hierten Substanz } \\
\text { + wässrigem Extrakt }\end{array}$} \\
\hline & & $i$ & $r$ & $i$ & $r$ \\
\hline & 80 & 1,94 & 82 & 1,5 & 75 \\
\hline Grau & 80 & 2, & 85 & 1,4 & 74 \\
\hline Weiss & 80 & 1,86 & 81 & 1,5 & 75 \\
\hline Welss (Vakuum) & $\begin{array}{l}72 \\
61\end{array}$ & $\begin{array}{l}1,63 \\
2,03\end{array}$ & $\begin{array}{r}86 \\
105\end{array}$ & $\begin{array}{l}1,08 \\
1.2\end{array}$ & $\begin{array}{l}72 \\
90\end{array}$ \\
\hline Foe"tus. & 78 & 1,77 & 82 & 1,4 & 76 \\
\hline
\end{tabular}

Die hemmende Wirkung ist also auf die weisse Substanz grösser als auf die graue; die Quellungsabnahme beträgt für die erstere im. Durehschnitt $18 \%$, für die letztere ca. $12 \%$. - Bei dem Gehirn des Fötus (90 cm lang) sind dieselben Einflüsse wie bei der grauen Substanz wirksam; die Hemmung ist geringer als bei der weissen. Da das unentwickelte Gehirn weniger Lipoide und mehr Proteine enthält, also in seiner Zusammensetzung der Rinde des erwachsenen Tieres ähnelt, wie auch die Analysen der Tabelle $V$ im Vergleich zu früheren Untersuchungen über die anorganischen Bestandteile zeigen, kann man folgern, dass die stärker hemmende Wirkung des Extraktes auf die weisse Substanz durch den grösseren Gehalt an Lipoiden bedingt ist. 
Die Quellung von Rinde und Treitungsbahnen des Grosshirns usw. 37

Die gewonnenen Resultate sind völlig eindeutig, da die Fehlerquelle für $i$ für die kleinsten Werte $\pm 0,05$, für die höchsten $\$ 0,60$ beträgt und für $r$ etwa 1,5 ausmacht.

Die angewandten Extrajtionsmittel haben alle die Eigenschaft, auf Eiweiss denaturierend, entquellend zu wirken, und durch Koagulation dio Struktur so zu verändern, dass das Quellungsvermögen herabgesetzi: wird. Diese Veränderung lrommt auch in der Kurve II in dem ständigen Fallen des Quellungsmaximums der grauen Substanz zum Ausdruck. Das Wasserbindungsvermögen der weissen Substanz dagegen steigt nach der Extraktion mit Benzol, also nach der Entfornung der Cerebroside, der P- und S-haltigen Galaktoside (s. S. 6) steil an. - Dieselbe Frscheinung beobachtet man, wenn man das trockene Gehirn direkt mit Benzol extrahiert und dann wioder das Quellungsvermögon prüft, wie die Zablen der Tabelle VIII beweisen.

Tabelle VIII.

Quellungsmaximnm der weissen Sabstanz nach Extraltion mit Benzol.

\begin{tabular}{c|c|c|c|c|c}
\hline Vorbehandlung & $\begin{array}{c}\text { Wasser- } \\
\text { gehalt der } \\
\text { lebenden } \\
\text { Substanz } \\
\%\end{array}$ & $\begin{array}{c}\text { Quellung vor der } \\
\text { Extraktion }\end{array}$ & $\begin{array}{c}\text { Quellung nach der } \\
\text { Extraktion }\end{array}$ \\
\hline $\begin{array}{c}\text { Mit Wasser und } \\
\text { Aceton extrahiert } \\
\text { (ufttrocken). }\end{array}$ & 61 & 0,89 & 77 & 1,0 & 82 \\
$\begin{array}{c}\text { Mit Wasser extra- } \\
\text { hiert (lufttrocken) }\end{array}$ & 72 & 1,18 & 74 & 1,22 & 77 \\
$\begin{array}{c}\text { Nicht extrahiert } \\
\text { (vakuumatrocken). }\end{array}$ & 72 & 1,44 & 82 & 1,63 & 86
\end{tabular}

Wenn man diesen Extraktionsprozess umkehrt, so findet man dasselbe Quellungsmaximum wie vor der Extraktion. Die Zahlen für diese Versuche ergeben sich aus Tabelle XI. Ich führte sio so aus, dass ich zu der völlig extrahierten weissen Substanz die im Vakuum eingeengten Extrakte hinzufügte und die so getränkte Masse bei $60^{\circ} \mathrm{C}$. im Vakuum trocknete. - Ċber die Deutung dieser Versuche siehe S. 0-.

\section{Die Quellungsgeschwindigkeit.}

Man hat in der Biologie oft Gesetzmässigkeiten, die man bei der Untersuchung relativ einfacher kolloider Systeme gefunden hatte, auf den lebenden Organismus übertragen, ohno zu bedenken, dass bei der hier herrsehenden Mannigfaltigkeit der stets weehselnden Kombinationen ganz andere Verhältnisse maassgebend sind, welche die gefundenen Tatsachen gerade in ihr Gegenteil verkehren können. 
Besonders oft findet man die Untersuchung zweier hydrophiler Gele, des Fibrins und der Gelatine in diesem Sinne angewandt. So schliesst zum Beispiel Fischer aus dem quellungsfördernden Einfluss verschiedener Salze und Säuren auf Fibrin auf einen ähnlichen Verlauf beim Ödem ${ }^{1}$ ), und Grober benutzte die bei dem Studium der Quellungsgeschwindigkeit der Gelatine in Gegenwart von Milchsäure gefundenen Veränderungen zur Erklärung der Muskelkontraktion ${ }^{2}$ ).

Wie wenig solche allgemeinen Übertragungen berechtigt sind, ergibt sich auch schon daraus, dass die Materialien, mit denen die einzelnen Forscher arbeiten, nie von einheitlicher Zusammensetzung sein können. Besonders beim Studium der Quellung bedingen die geringsten Unterschiede im Elektrolytgehalt andere Gesetzmässigkeiten. - Für die Quellungsgeschwindigkeit stellte zuerst Hofmeis ter eine Formel auf ${ }^{3}$ ), welche die Abhängigkeit von der chemischen Beschaffenheit der quellenden Substanz, von der Dicke der Schicht und dem Quellingsmaximum berücksichtigt; nach einer einfachen Umformung lautet diese: $\frac{c}{d}=\frac{1}{t} \cdot \frac{x}{a-x}$, wobei $c$ eine Konstante bedeutet, die von der chemischen Natur der Substanz abhängig ist, $d$ die Schichtdicke, $t$ die Zeit, $a$ das Quellungsmaximum und $x$ den Quellungsgrad zur Zeit $t$. - Pauli stellte die Differentialgleichung auf: $\frac{d x}{d t}=K \cdot(a-x)$, ohne den Einfluss der Schichtdicke, wie sie bei den gewähiten Versuchsobjekten Hofmeisters, Gelatinescheiben, berücksichtigt werden muss, zu beachten ${ }^{4}$ ). Schröder führte diese Formel unter Berücksichtigung dieses Faktors über in $\frac{d x}{d f}=k^{1} \cdot \frac{a-x}{x \cdot w}$, wobei $w$ den mittleren Quellungsgrad (Wassergehalt) der untersuchten Gelatine in jedem Moment bedeutet ${ }^{1}$ ). - Er gibt aber keinen Weg zur Integration dieser Gleichung an, indem er die Funktion zwischen $x$ und $w$ entwickelt. Folgende UUberlegung führt hier vielleicht zum Ziel: Denkt man sich die quellende Schicht in unzählige kleinere Schichten zerlegt, von denen jede den mittleren Quellungsgrad $w_{1}$ haben möge, den man bei der geringen Dicke gleich der gebundenen Wassermenge $m$ setzen kann, so ist die Summe aller dieser einzelnen Mengen gleich der von der ganzen Schicht

1) M. H. Fischer, Das Ödem. Dresden 1910.

2) J. Grober, Muskelkontraktion und Kolloidquellung. Münch. med. Wochenschr. 1912 S. 2433.

3) F. Hofmeister, Untersuchungen über den Quellungsvorgang. Arch. f. exper. Pathol, u. Pharmakol. Bd. 27 S. 402.1890.

4) W. Pa scheles, Versuche über die Quellung. Pflü ger's Arch. Bd. 71 S. 347.1898.

5) P. v. Schroeder, Erstarrungs- und Quellungserscheinungen von Gelatine. Zeitschr. f. physik. Chem. Bd. 45 S. 111. 1903. 
Die Quellung von Rinde und Leitungsbahnen des Grosshims usw. 30

gebundenen $x$, und da der mittlere Wassergehalt gleich der Summe aller dieser kleinen Mengen dividiert durch die Schichtdicke ist, so ergibt sich: $\frac{\sum m}{d}=\dot{w}=\frac{x}{d}$. Setzt man diesen Wert für $w$ in die obige Gleichung cin, so folgt: $\frac{d x}{d t}=k \cdot d \frac{a-x}{x^{2}}=k \cdot \frac{a-x}{x^{2}}$, - Nach Integration dieser Gleichung, die ich mit liebenswürdiger Unterstützung des Herm Dr. Eichwald ausführte, ergibt aber auch hier die Berechnung der Konstanten mit der Zeit steigende Werte, obenso wie die Anwendung der Pauli'schen Gleichung auf dic versehiedensten Beispielo, die ich der Schröder'schen Arbeit, Quellungsversuchen von Hofmeister und lischer und Sykes entnahm, fallende Konstanten orgibt ${ }^{\mathbf{1}}$ ). - Bei dor letzteren, Quellung von Gelatine in Wasser, stimmen dagegen die Konstanten der Schütz'schen Gleichung ziemlich überein, während sie bei den Vorsuchsreihen der beiden anderen Autoren ebenfalls fallen, obenso wie bei der Durchreshnung meiner Tabellen - ausgenommen Tab. X, I - Woiss mit den Konstanten: 0,41 $0,38-0,38-0,41-0,46$. Tabelle IX gibt die nach Fischer (1. c. S. 218) aus ciner bolicbig gewählten Versuchsreihe berechneten verschiedenen Konstanten wieder. '- Freundlich berechnet die Quellungsgeschwindigkeit nach der Formel $k=\frac{1}{t-t_{1}} \ln \frac{a}{a}-\frac{x}{-x_{1}}$, abor auch in dem von ihm angegebenen Gelatineversuch fallen die Konstanten, im Gegensatz zu einer Quellung mit einem Pflanzengel (Agar) ${ }^{2}$ ).

Tabelle IX.

Die Konstanten für die Gleichungen der Quellungsgeschwindigkeit. Berechnet für die Quellung von Gelatine in Wasser (Fischer und Sykes $k_{m}=\frac{1}{t} \cdot \ln \frac{a}{a-x}=$ Konstante der Pauli'schen Gleichung, $k_{s}-\frac{x}{\sqrt{t}}=$ Konstante der Schütz'schen Gleichung, $k_{s c h}=\frac{1}{t}\left(\ln \frac{a}{a-x} \cdot a^{2}-\frac{x^{2}}{2}-a \cdot x\right)=$ Schroeder'sche Konstante. (km mit dekadischen, $k_{s c h}$ mit natürlichen Isogarithmen berechnet.)

\begin{tabular}{c|c|c|c|c|c}
\hline$t$ & $x-2=i$ & $a-x$ & $k_{m} \cdot 10^{4}$ & $k_{8} \cdot 10^{2}$ & $l_{s m} \cdot 10$ \\
\hline 7,0 & 2,90 & 7,59 & 202 & 110 & 13 \\
22,3 & 6,04 & 4,39 & 169 & 128 & 59 \\
31,0 & 6,77 & 3,66 & 146 & 122 & 66 \\
46,3 & 8,44 & 1,99 & 155 & 124 & 139
\end{tabular}

1) M. H. Fischer u. A. Sykes, Über den Finfluss einiger Nichtelektrolyte auf die Quellung von Proteinen. Kolloidzeitschr. Bd. 14 S. 215. 1914.

2) H. Freundlich, Kapillarchemie 1909 S. 510. 


\begin{tabular}{c|c|c|c|c|c}
\hline$t$ & $x=i$ & $a-x$ & $k_{m} \cdot 10^{4}$ & $k_{s} \cdot 10^{2}$ & $k_{s c h} \cdot 10$ \\
\hline 55,0 & 8,77 & 1,66 & 145 & 118 & 129 \\
71,45 & 9,42 & 1,01 & 142 & 111 & 156 \\
79,15 & 10,43 & 0 & - & 117 & -
\end{tabular}

Schon Hofmeister selbst hat auf diese Unstimmigkeiten hingewiesen und gefunden, dass die von ihm gegebenen Formeln keine übereinstimmenden Konstanten zeigten. - Er suchte diesen Fehler auszugleichen, indem er die dureh Berechnung zu findende Zahl $F$ von der oben angeführten Gleichung subtrahierte ${ }^{1}$ ). - Die Erscheinung der fallenden monomolekularen Konstanten, wie sie Tabelle IX andeutet $\left(k_{m}\right)$, und wie ich sie bei der Durchrechnung anderer Beispiele noch ausgeprägter fand, deutet in Analogie mit anderen Adsorptionserscheinungen darauf hin, dass die Reaktion durch irgendeinen Umstand dauernd verzögert wird ${ }^{2}$ ). Als Ursache für diese Reaktionshemmung kann man bei der untersuchten Gelatine den wechselnden Gehalt an anorganischen Bestandteilen annehmen, deren quellungsfördernde oder -hemmende Wirkung bei stark geouollener Gelatine eine andere ist, als bei geringem Wassergehalt, so dass zum Beispiel die lyotrope Anionenreihe umgekehrt wird (vgl. S. 3). - Freundlich erklärt die Hemmung durch die ungleichmässige Durchdringung, so dass die äusseren Schichten schneller das Maximum erreicht haben als die inneren ${ }^{3}$ ).

Bei der Bestimmung der Zeitkurve für die graue und weisse Substanz galt es also, alle diese Faktoren zu berïcksichtigen. Am meisten Schwierigkeiten machte es, die Schichtdicken in Übereinstimmung zu bringen. Die Art der Präparation bedingt es, dass man die graue Substanz nur in dünnen, ungleichmässigen Schichten gewinnen kann, während es bei der weissen sehr gut gelingt, gleichmässig dicke Streifen zu erhalten. -- Ich suchte diese Unterschiede dadurch auszugleichen, dass ich gleiche Mengen der trockenen Substanzen abwog, die ich dann zerbröckelte oder in möglichst gleich grosse Stückchen zerschnitt. - Der Einfluss der nicht adsorbierten Elektrolyte wurde dadurch ausgeschaltet, dass ich meistens nur die zweite Quellung zur Bestimmung der Kurvenpunkte benutzte oder vorher einen Tag durch destilliertes Wasser, das öfter erneuert wurde, bei Zimmertemperatur die zu untersuchenden Mengen auslaugte. - Wie ich schon oben erwähnte, arbeitete ich mit grösseren Serien und bestimmte

1) 1. c. VI. Mitt. Arch. f. exper. Path. u. Pharmak. Bd. 28 S. 219. 1891.

2) A. Fod or, Handbuch d. biochem. Arbeitsmethoden Bd. IX S. 55̌. 1919.

3) 1. c. S. 511. 
Die Quellung von Rinde und Leitungsbahnen des Grosshirns usw. 41

die verschiedenen Quellungsgrade nicht an ein und demselben Stück. Wenn die Versuchsfehler unter diesen rohen Bedingungen auch recht grosse sein können, so ergaben doch alle Untersuchungen eindeutig den Einfluss der chemischen Struktur auf die Geschwindigkeit der Quellung. Die grave Substanz erreichte schneller das Quellungsmaximum als die weisse; bei der zweiten Quellung ist die stäkere Quellung der letzteren nach dem Ausschalten des hemmenden Einflusses der wasserlöslichen Bestandteile deutlich erkennbar. - Um diesen Unterschied zwischen der Quellungsgeschwindigkeit der Rinde und Leitungsbahnen aufzulalären, untersuchte ich die Zeitkurven bei den verschiedenen Fraktionen, die nach der Extraktion zurïckblieben und verglich sie mit von homogenem Eiweiss und Lipoiden gewonnenen. Um zu zeigen, dass die typischen Unterschiede, die ich hierbei fand, nicht durch die verschiedene Schichtdicke bodingt sind, sondern eine Funktion der chemischen Struktur darstellen, wählte ich zunächst als Versuchsmaterial Serum- und Plasmaeiweiss, das ich in dünner Schicht (Oxalatblut) in Kristallisierschalen bei $100^{\circ} \mathrm{C}$. gerinnen liess, so dass ich gleichmässig dicke Streifen herausschneiden konnte; dann die verschiedenen Extrakte, die beim Eindampfen im Vakuum zu glasigen, leicht schneidbaren Massen erstarrten. - Ferner variierte ich die Schichtdicke und nahm grobe Stücke des geronnenen Eiweisses, deren Quellungskurve ich mit der von kristallisiertem Cholesterin verglich, das aus den Acetonextrakten ausgefallen war. - Aber auch hier zeigte sich noch das typische Verhalten der Proteine, schon nach kurzer Zeit das Quellungsmaximum zu erreichen, während die Kurve der Lipoide nicht so schnell anstieg. - Hiermit wird auch das verschiedene Verhalten der grauen und weissen Substanz erklärt: der höhere Lipoidgehalt der letzteren bedingt den langsamen Verlauf der Quellung im Gegensatz zu der eiweissreicheren Rinde. Nach der vollständigen Extraktion ist dieser Unterschied im Verlauf der Kurven verwischt; es zeigt sich der Typus der Proteinquellung mit raschem Anstieg. - Zusätzo der verschiedenen Extrakte gaben keine eindeutigen Resultate; sie zeigen aber die Wiedererhöhung des Quellungsmaximums (vgl. S. 16).

Um möglichst einwandfreie Zahlen zu erhalten, wählte ich Stundenabstände, da so die relativen Quellungsgrade eine genügend grosse Differenz haben, um die Fehlergrenze $(1,5)$ ausschalten zu können. In der Tabelle $\mathrm{X}$ gebe ich auch eine Versuchsreihe mit luft rockenem Material wieder, bei der aber die Unterschiede nicht so gut zu erkennen sind wie bei dem rakuumtrockenen. Die Frage, ob es sich bei dieser verschiedenen Quellungsart um ein rein additives Verhalten von Proteinen und Lipoiden handelt, ist auf diesem Wege nicht zu lösen; der Verlaur der Kurven lässt ebenso gut diese Deutung zu, wie 
die Annahme neuer Adsorptionsverbindungen, die eine andere Quellungskurve besitzen als die einzelnen Komponenten.

Tabelle X.

Quellungsgeschwindigkeit der grauen und weissen Substanz.

Rindergehirn. I lufttrocken, II vakuumtrocken. Zahlen $=i$.

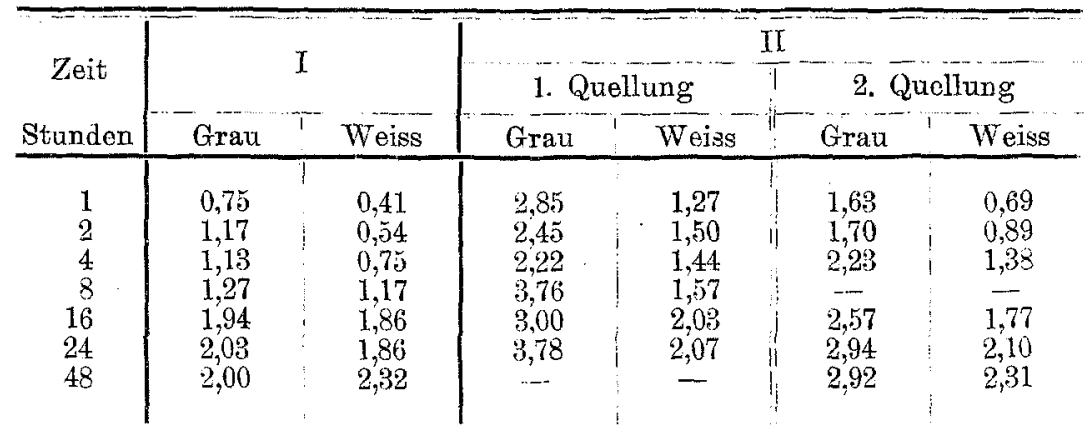

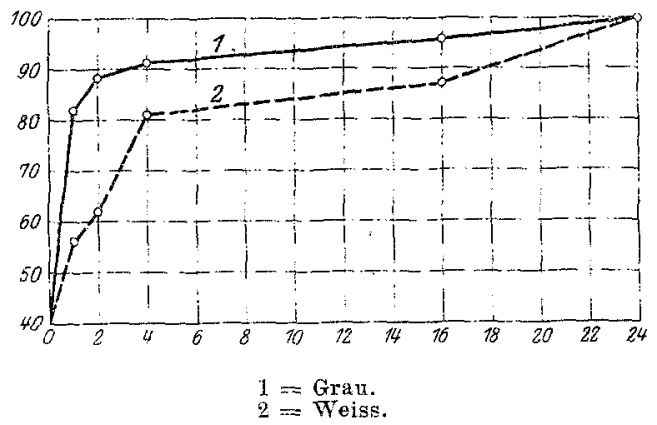

Abb. 3. Quellungsgeschwindigkeit während der zweiten Quellung. Rindergehirn. Vakuumtrocken $60^{\circ}$. Abszisse $=$ Zeit in Stunden. Ordinate $=$ Prozente des maximalen Wassergehalts nach 24 Stunden.

Tabelle XI.

Quellungsgeschwindigkeit nach fraktionierter Extraktion.

Zahlen $=-i$.

\begin{tabular}{|c|c|c|c|c|c|}
\hline Zeit & $\begin{array}{c}\text { Weiss } \\
\text { mit Benzol } \\
\text { extrahiert }\end{array}$ & $\begin{array}{c}\text { Völlig } \\
\text { Grau }\end{array}$ & $\begin{array}{l}\text { hiert } \\
\text { Weiss }\end{array}$ & $\left|\begin{array}{c}\text { Vollig } \\
\text { extrahiert } \\
+ \text { Petrol- } \\
\text { atherextra.kt }\end{array}\right|$ & $\begin{array}{l}\text { Weisse } \\
\text { Substanz } \\
+\quad \text { Alkohol- } \\
\text { extrakt }\end{array}$ \\
\hline $\begin{array}{l}1 \\
2 \\
4 \\
8\end{array}$ & $\begin{array}{l}1,38 \\
1,75 \\
2,57\end{array}$ & $\begin{array}{l}0,98 \\
1,08 \\
1,17 \\
1,19\end{array}$ & $\begin{array}{l}0,73 \\
1,17 \\
1,24 \\
1,27\end{array}$ & $\begin{array}{l}1,12 \\
1,20 \\
1,25 \\
1,25\end{array}$ & $\begin{array}{l}0,89 \\
1,26 \\
1,36 \\
1,38\end{array}$ \\
\hline
\end{tabular}


Die Quellung von Rinde und Leitungsbahnen des Grosshirns usw. 43

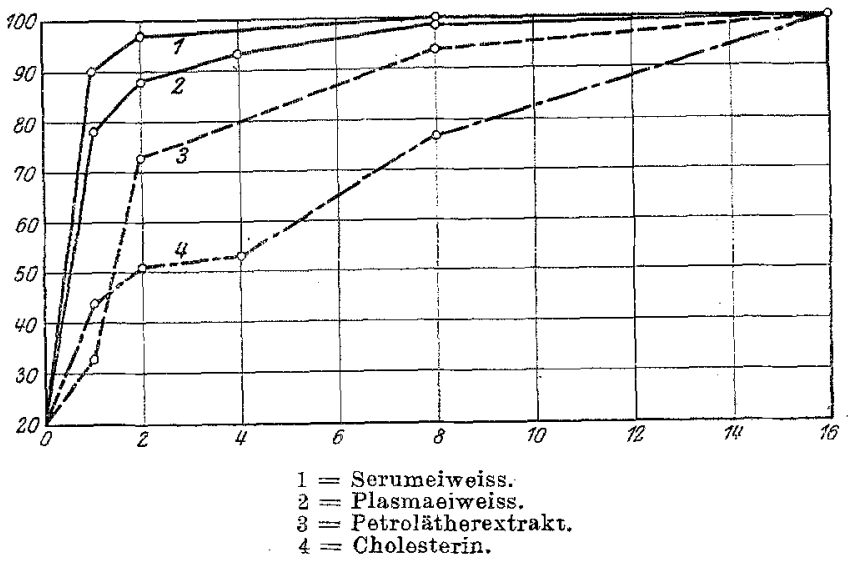

Abb. 4. Quellungsgeschwindigkeit von Proteinen und Lipoiden. Abszisse $=$ Zeit in Stunden. Ordinate $=$ Prozente des maximalen Wassergehalts nach 16 Stunden.

\section{Einfluss der Temperatur auf die Quellung.}

In der mir zur Verfügung stehenden Literatur konnte ich keino systematischen Untersuchungen über den Einfluss der Temperatur auf das Wasserbindungsvermögen der hydrophilen Kolloide finden. Die meisten Autoren erwähnen nur kurz, dass bei steigender Temperatur der Quellungsgrad zunehme, ohne nähere Angaben zu machen. Wenn man die Quellung als Adsorptionserscheinung oder als konzentrierte Lösung des Wassers in dem quellenden Körper auffasst, so muss man nach allen hierbei in Betracht kommenden Gesetzen eine Vermehrung des gebundenen Wassers mit steigender Temperatur erwarten. - Die Versuche, die ich zunächst an einfachen Systemen, denselben wie beim Studium der Geschwindigkeit ausfithrte, bestätigten diese theoretische Voraussetzung: Die Temperaturkurve steigt mit zunehmender Wärme. - Um so überaschender war die Abweichung von diesen Gesetzmässigkeiten bei der Quellung der grauen und weissen Substanz: Bei Körpertemperatur erreicht die Kurve ein Minimum, um dann wieder anzusteigen. Bei allen untersuchten Rindergehirnen fand ich diese Erscheinung wieder, bald mehr, bald weniger ausgesprochen; niemals aber war der Quellungsgrad bei $40^{\circ} \mathrm{C}$. höher als bei Zimmertemperatur. Da während der letzten Monate der Gasdruck dauernd wechselte, war es nicht möglich, die Thermostaten bis auf $1^{\circ} \mathrm{C}$. Konstant zu erhalten; die Zahl 20 in den folgenden Tabellen bedeutet daher Temperaturintervalle von $18-21^{\circ}$, die Zahl 40 ist durch $36-40^{\circ}$ zu ersetzen. Die Zu- und Abnahme der relativen Quellungszahlen innerhalb $5^{0}$ liegt aber zwischen den Fehlergrenzen, so dass feinere Untersuchungen an Gehirnsubstanz zwecklos sind. 
Die Differenz von 20 Graden zwischen den einzelnen Kurvenpunkten gibt dagegen eindeutige Resultate. - Auch bei der Rinde und weissen Substanz eines etwa 8 Monate alten Fötus fand ich dieselbe Temperaturkurve. Dagegen zeigt das völlig extrahierte Gehirn wieder den Typus der anderen, einfacheren Gele. - Man kommt bei dein Versuch, diese Abweichungen zu erklären, nicht mehr mit der Annahme einer reinen Addition der Eigenschaften von Proteinen und Lipoiden in dem quellenden System Gohirn aus, sondern man ist zu der Annahme gezwungen, dass beide eine neue Verbindung gebildet haben, die völlig andere Eigenschaften besitzt als die einzelnen Bestandteile. Eine zwcite Erklärung giht die schon erwähnte, ron Lenk gefundene Umkehrung der lyotropen Reihe mit zunehmender Quellung; die Lyotropie der quellenden Teilchen und die Quollungshemmung der Eloktrolyte würden danach mit steigender Temperatur antagonistisch wirken, so dass als Resultante dieser entgogengesetzten Kräfte beim Überwiegen der letzteren eine Quellungsabnahme entstände, im Sinne neuerer Theorien eine Verdrängung von Wasser von der Oberfläche der Kolloide. - Alle diese Hypothesen bedürfen noch der Klärung durch das Studium der Einwirkung kombinierter Salzgemische auf die Quellung kolloider Systeme bei verschiedenen Temperaturen.

Es scheint, als ob der erneute Anstieg der Quellung sehon unterhalb $40^{\circ}$ beginnt, doch konnte ich aus den oben angeführten Gründen diese feineren Differenzierungen noch nicht weiter verfolgen. - Zum Vergleich herangezogenes Pferdegehirn, bei dem die Unterschiede im Wassergehalt von Grau und Weiss $(80$ und $66 \%$ ) grösser sind, als beim Rinde ( 80 und $69 \%$ ), wohl infolge des grösseren Lipoidgehaltes der Leitungsbahnen, beobachtete ich dieselben Quellungsunterschicde. Ein zur Orientierung vorgenommener Extraktionsversuch ergab für das Pferdegehirn an benzollöslichen Lipoiden: Grau 39\%, Weiss $63 \%$ der 'Trockensubstanz; für das Rindergehirn: Grau $35 \%$, Weiss $59 \%$. -Hiernach ist die bei den verschiedenen Tierarten gefundene Differenz zwischen dem wechselnden Wassergehalt von Rinde und Leitungsbahnen wohl abhängig von dem Untersehied im Lipoidgehalt; je grösscr dieser Unterschied sein wird, desto mehr Wasser wird auch die graue Substanz im Verhältnis zur weissen enthalten. - Die bei der fraktionierten Extraktion erhaltenen Ergebnisse sind die beste Stütze für diese Auffassung.

Auf die Quellungsgeschwindigkeit hat die Temperaturerhöhung wenig Einfluss; die Unterschiede zwischen Grau und Weiss sind dieselben wie bei $20^{\circ}$. - Die folgenden Tabellen geben die Durchschnittszahlen grösserer Versuchsreihen wieder; bei dem lufttrockenon Gehirn von 22, bei dem zweiten und dem fötalen von 14 Einzelverstuchen. 
Die Quellung von Rinde und Leitungsbahnen des Grosshirns usw. 45

Tabelle XII.

Finfluss der Temperatur auf die Quellung. Zahlen $=i$.

\begin{tabular}{r|c|c|c|c|c|c}
\hline $\begin{array}{c}\text { Tempe- } \\
\text { ratur }\end{array}$ & $\begin{array}{c}\text { Rind I } \\
\text { lufttrocken }\end{array}$ & \multicolumn{2}{c|}{$\begin{array}{c}\text { Rind II } \\
\text { vakuumtrocken }\end{array}$} & \multicolumn{2}{c}{$\begin{array}{c}\text { Foetus } \\
\text { ca. Monate }\end{array}$} \\
\hline $0^{\circ}$ & Grau & Woiss & Grau & Weiss & Grau & Weiss \\
$20^{\circ}$ & 1,90 & 1,32 & 2,69 & 1,59 & 2,22 & 1,34 \\
$40^{\circ}$ & 1,09 & 1,45 & 2,57 & 2,21 & 2,15 & 1,90 \\
$60^{\circ}$ & 2,32 & 1,24 & 2,25 & 1,65 & 1,86 & 1,08 \\
& 1,49 & 3,75 & 2,44 & 2,40 & 1,86
\end{tabular}

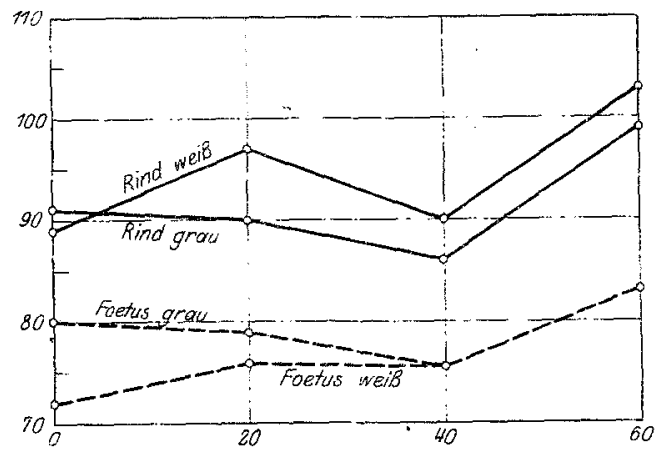

Abb. 5. Temperatur und Wasseraufnahme bei der zweiten Quellung. Abszisse -... 'Temperatur. Ordinate $=r$-Prozente des Wassergehalts der lebenden Substanz.

Tabelle XITI.

Einfluss der Temperatur anf die Quellung. Zahlen $=i$.

\begin{tabular}{|c|c|c|c|c|c|c|}
\hline \multirow{2}{*}{$\begin{array}{l}\text { Tempe- } \\
\text { ratur }\end{array}$} & \multicolumn{2}{|c|}{$\begin{array}{l}\text { Völlig extrahierte } \\
\text { Substanz (Rind) }\end{array}$} & \multirow[t]{2}{*}{ Plasma } & \multirow[t]{2}{*}{ Serum $^{1}$ ) } & \multirow{2}{*}{$\begin{array}{l}\text { Chole- } \\
\text { sterin }\end{array}$} & \multirow{2}{*}{$\begin{array}{l}\text { Petrol- } \\
\text { äther- } \\
\text { extrakt }\end{array}$} \\
\hline & Grau & Weiss & & & & \\
\hline $\begin{array}{l}0^{\circ} \\
20^{\circ} \\
40^{\circ} \\
60^{\circ}\end{array}$ & $\begin{array}{l}1,04 \\
1,13 \\
1,27 \\
1,63\end{array}$ & $\begin{array}{l}1,23 \\
1,15 \\
1,33 \\
1,70\end{array}$ & $\begin{array}{l}2,12 \\
2,45 \\
2,85 \\
4,25\end{array}$ & $\begin{array}{l}1,86 \\
2,03 \\
2,45 \\
9,0\end{array}$ & $\begin{array}{c}\overline{2,21} \\
3,76 \\
13,6\end{array}$ & $\begin{array}{l}1,03 \\
1,08 \\
1,77 \\
2,45\end{array}$ \\
\hline
\end{tabular}
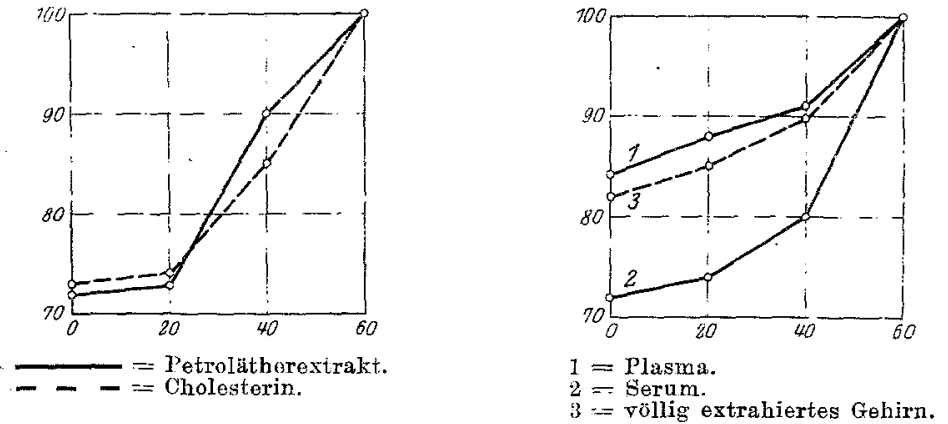

Abb.6u. 7. Finfluss der Temperatur auf die Wasseraufnahme bei Proteinen und Lipoiden. Abszisse $=$ Temperatur. Ordinate $=$ Proz. d. Quellungsgrades bei $60^{\circ}$. 1) Iufttrocken. 
Die Bedeutung des Quellungsminimums bei Körpertemperatur für die Regulierung der Lebenstorgänge an dieser Stelle zu diskutieren, würde über den Rahmen der Arbeit hinausgehen. Nur auf eine beim Studium der elektrischen Vorgänge in den peripheren Nerven gefundene Abweichung von der Membrantheorie möchte ich noch die Aufmerksamkeit lenken, um vielleicht einen Weg zu zeigen, der zn nenen Erklärungsmöglichkeiten führt.

An isolierten Nerven kann man beimi Erwärmen und Abkühlen von Längs- und Querschnitt elektrische Ströme ableiten. Nach Verzàr ${ }^{1}$ ) ist zwischen 0 und $20^{\circ}$ hierbei die wärmere Stelle positiv gegen die kältere; ,oberhallb $20^{\circ}$ dagegen ist das Verhalten des Längsschnittes im höchsten Grade unregelmässig. Manchmal ist auch hier die warme Stelle positiv, oder die Änderungen sind verschwindend klein, oder es ist auch öfters die warme Stelle negativ gegen die kältere, also das Gegenteil des Ergebnisses der Kühlungsversuche." Er glaubt dieses unregelmässige Verhalten damit erklären zu können, dass der Nerv bei der höheren Temperatur geschädigt würde und dadurch abstürbe; seine eigenen Versuche widerlegen aber diese Auffassung, da die Erscheinungen beim wiederholten Abkühlen und Wiedererwämmen reversibel sind. Bernstein, auf dessen Veranlassung diese Versuche unternommen wurden, nimmt als Ursache eine mit der Temperatur wechselnde Konzentration der Elektrolyte in der Nervenfaser an, ,trotzdem diese Erklärung auch noch sehr unwahrscheinlich ist, da die Inderung der Kraft mit wechselnder Temperatur immer reversibel ist und ein so schnelles Verschwinden entstandener Elektrolytmengen nicht möglich ist" ${ }^{2}$ ). Als Ursprungsstelle der Thermoströme wird im Sinne der Membrantheorie nach Cremer ${ }^{3}$ ) am Querschnitt die geschlossene Membran angesehen, welehe die Ranvier'sehen Segmente umgibt und am Längsschnitt die äusseren Hüllen des Nerven ${ }^{4}$ ). Wenn man die bioelektrischen Ströme nach Bernstein als Diffusionspotentiale auffasst, so ist die Umkehrung der Richtung beim Erwärmen über $20^{\circ}$ nicht erklärbar. Nimmt man dagegen Adsorptionspotentiale ais Ursache an 5), -- eine Annahme, die wohl am besten mit dem Quellungszustande der hydrophilen Kolloide des Nervensystems vereinbar ist - , so ist es leicht verständlich, dass mit der Änderung des Adsorptionsvermögens der Systeme Ejweiss-Lipoide bei steigender Temperatur die Richtung der Ströme umgekehrt werden muss, da ja die

1) F. V erzàr, Über die Natur der Thermoströme des Nerven. Pf1üger's Arch. Bd. 143 S. 252.1912.

2) J. Bernstein. Elektrobiologie S. 102. Braunschweig 1912.

3) Nach Verzàr, l. c. S. 280.

4) A. v. Tschermak, Julius Bernstein's Lebensarbeit. Pflüger's Arch. Bd. 174 S. 1. 1919.

5) R. Höber, 1. c. S. 244. 
Die Quellung von Rinde und Leitungsbahnen des Grosshims usw. 47

Temperaturerhöhung über $20^{\circ}$ dieselben Veränderungen an der adsorbierenden Oberfläche hervorrutt wie die Abkühlung von 20-0 . Bei Quellungszunahme (Erwärmen von 0-200 oder Abkühlen von 40-20\%) ist die wärmere Stelle positiv gegen die kältere; bei Quellungsabnahme (Abkühlen von $20-0^{\circ}$ oder Erwärmen von $20-40^{\circ}$ ) dagegen ist die wärmere negativ gegen die kältere.

Gegen die Auffassung, dass die bioelektrisohen Ströme durch Diffusionspotentiale entstehen, sind anch sonst die verschiedensten Einwände erhoben worden. - Neuere Theorien gehen dahin, die Potentialdifferenzen auf Kräfte zurückzuführen, die an der Grenze zweier Phasen die Konzentration der vorhandenen Ionen danernd ändern, sei es nun dadurch, dass Anionen und Kationen in den beiden Phasen verschieden löslich sind, mit ihnen verschiedene chemische Bindungen eingehen, wie in den Modellen Beutner's ${ }^{3}$ ) oder an die kolloiden Phasen verschieden stark adsorbiert werden, wie beim Entstehen ion Grenzpotentialen zwischen Adsorbens und Eleiktrolytlösungen im Sinne Freundlich's ${ }^{2}$ ).

In den Leitungsbahnen des Nervensystems kämen als Grenzphasen zum Beispiel Achsenzylinderfortsatz und Markscheide oder Fibrillen und interfibrilläre Substanz in Frage. Da beide Phasen chemisch völlig differenziert sind und inr Quellungsvermögen durè den wechselnden Gehalt an Proteinen, Lipoiden und Elektrolyten ebenfalls verschieden ist, so ist es nach den obigen Theorien verständlich, dass an der Grenze dieser beider Systeme Potentialdifferenzen auftreten müssen, die als Ruheströme nachgewiesen werden können. Boruttau entwickelte auf Grund dieser Verschiedenheit von Neurofibrillen und Achsenzylinder seine Kernleitertheorie, die eine Polarisation an der Grenzläche beider annimmt ${ }^{3}$ ). - Diese Hinweise mögen genügen, um die Bedeutung der nntersuohten Quellungsunterschiede zwischen den verschiedenen Phasen des Nervensystems für die Entistehung der bioelektrisohen Ströme hervorzubeben.

Wie Katz ${ }^{4}$ ) wieder bewies, ist die Quellung der Gele mit dem Treiwerden grosser Energiemengen verbunden, die als Quellungswäme nachgewiesen werden können. Daduroh, dass bei Körpertemperatur die nervösen Systeme auf ein Quellungsminimum eingestellt sind, ist stets eine bestimmte Menge Wärme als latente Energie gebunden, sc dass wir auch in dem Quellungsminimum eine „Vitaldifferenz" mit

1) R. Beutner, Zeitschr. f. Elektrochemie Bd. 19. 1913.

2) H. Freundlich u. Mäkelt, ebenda Bd. 15 S. 161. 1909. Zitiert nach $\mathrm{H} \ddot{\mathrm{o}} \mathrm{b} \in \mathrm{r}, \mathrm{1}$, c.

3) Vgl. hierzu M. Verw orn, Die Vorgänge in den Elementen des Nervensystems. Zeitschr. f. allgem. Physiol. Bd. 6 S. 42.1907.

4) 1. c. S. 172 . 
ständiger Arbeitsbereitschaft vor uns haben. Ob allerdings die bei weiterer Quellung -.. sei es durch Anhäufung von Stoffwechselendprodukten oder Zerfallsstofien bei pathologischen Prozessen - auftretende Wärmemenge bei dem relativ hohen Quellungsgrade noch gross genug ist, um messbar als Fieber nachgewiesen werden zukönnen, müsste durch besondere Studien geprüft werden.

Die Bedeutung der Quellung für die Lebensvorgänge im allgemeinen hat Pribram ${ }^{1}$ ) in einer grösseren Arbeit zusammengestellt. Eine allzu starke Betonung des Einflusses der Quellung würde aber ebenso zurückzuweisen sein, wie andere einseitige Theorien, die durch eine Möglichkeit das ganze komplizierte System erklären wollen, in dem in grösster Mannigfaltigkeit die verschiedensten Prozesse ineinandergreifen and sich gegenseitig beeinflussen. - Auch der Wassergehalt des Nervensystems ist eine Funktion, die nicht allein von einer Veränderlichen, der chemischen Struktur, abhängig ist, sondern auch gleichzeitig von allen anderen Organen des lebenden Organismus, die durch Vermittlung des Blutes und der Nervenbahnen die Quellung beeinflussen. In einer kurzen Zusammenstellung will ich versuchen, die Bedeutung dieses ersten Faktors aufzudecken, um so gleichzeitig noch einmal die in dieser Arbeit gewonnenen Ergebnisse zusammenzufassen.

\section{Allgemeine Folgerungen.}

1. Die Lufttrocknung von Nervengewebe bedingt eingreifende Veränderungen der chemischen Struktur, die alle theoretischen, aus Versuchen an so präpariertem Material geschlossenen Folgerungen wertlos machen. Die Trocknung im Faust-Heim'schen Apparat und im Trockenschrank ist daher zu verwerfen.

2. Die von den Lipoiden nicht zu trennenden anorganischen Bestandteile sind keine "Verunreinigungen", sondern bilden mit diesen einheitliche Körper vom Typus der Adsorptionsverbindungen, ebenso wie Proteine und Lipoide nicht nebeneinander und unabhängig voneinander im Nervensystem vorhanden sind, sondern ebenfalls festere Verbindungen eingehen, die aber nicht in stöchiometrischen VerhäItnissen durch chemische Bindung erfolgen, sondern von Oberflächenkxäften abhängen, vielleicht in der Art, dass durch verschiedene elektrische Aufladung der einzelnen Teilchen durch die Ionen der adsorbierten Elektrolyte und des Quellungswassers eine gegenseitige Anziehung erfolgt.

1) E. Přibram, Die Bedeutung der Quellung und Entquellung fur physiologische und pathologische Erscheinungen. Kolloidzeitschr. Beihefte. B d. 2 S. 1. 1910. 
Die Quellung von Rinde und Leitungsbahnen des Grosshirns usw. 49

3. Die von Hofmeister, Pauli und von Sçhroeder aufgestellten Formeln für die Quellungsgeschwindigkeit haben nach Prüfung der Konstanten an den verschiedensten, aus der Literatur entnommenen Beispielen keine allgemeine Gültigkeit; in einzelnen Fällen scheint $k=\frac{i}{\sqrt{t}}$ zu sein, während die Konstanten der beiden ersten Formein stetig fallen, ein Ausdruck dafür, dass mit steigendem Quellungsgrade die hemmende Wirkung nicht berücksichtigter Faktoren, wahrscheinlich der stets vorhandenen Elektrolyte, zunimmt.

4. Die Quellungsgeschwindigkeit der Proteine ist grösser als die der untersuchten Lipoide.

5. Mit steigender Temperatur nimmt der Quellungsgrad beider zu.

\section{Die Quellung der granen und weissen Substanz.}

6. Die Proteine der Ganglienzellen und der Achsenzylinder der Leitungsbahnen haben in bezug auf Elemente und Aminosäuren die gleiche Zusammensetzung; auch eine Strukturisomerie ist aus histiologischen und entwicklungsgeschichtlichen Gründen wahrscheinlich.

7. Die im Nervensystem vorhandenen Kombinationen von Eiweiss und Lipoiden bedingen einen hohen Widerstand gegen die künstlich gesteigerten, das Quellungsvermögen schädigenden Einflüsse der wiederholten Quellung und Entquellung (Hysteresis), also auch gegen die Wirkungen des steten Wechsels des Quellungsgrades während des Lebens.

8. Der geringere Wassergehalt der Leitungsbahnen wird bedingt:

a) durch die grössere quellungshemmende Wirkung extrahierbarer, wasserlöslicher Bestandteile auf die weisse Substanz;

b) durch den grösseren Gehalt an benzollöslichen Lipoiden, die ebenfalls das Wasserbindungsvermögen der Proteine beeinflussen.

9. Die Quellungsgeschwindigkeit der Rinde ist grösser als die der Leitungsbahnen, da sie mehr Proteine enthält.

10. Die Quellung nimmt nicht mit steigender Temperatur $\mathrm{zu}$ wie bei den isolierten Proteinen und Lipoiden, sondern erreicht bei etwa $20^{\circ} \mathrm{C}$. ein Maximum, um dann bis etwa $36^{\circ} \mathrm{C}$. wieder zu sinken. Diese Erscheinung dient zur. Erklärung des Richtungsumschlages der Thermoströme im, Nerven bei Temperaturen über $20^{\circ} \mathrm{C}$. 